\title{
MODELO DE PREDIÇÃO DA ACESSIBILIDADE E MOBILIDADE URBANA
}

\author{
André de Souza Silva ${ }^{1}$
}

\begin{abstract}
RESUMO
Apresentar os resultados obtidos com o desenvolvimento do Modelo de Deslocamento Sustentável em Cidades, indicar as possibilidades de continuidade da pesquisa e contribuir com sugestões para futuros estudos são os objetivos deste artigo. Trata-se de um modelo de predição que possibilita a análise abrangente do movimento de pedestres e veículos, uma vez que simula alternativas de decisões de desenho urbano e verifica a influência dos regimes urbanísticos no planejamento de áreas novas e preexistentes. Operacionalmente viável e promissor, o modelo matemático apresenta resultados estatísticos significativos e com alto grau de confiabilidade. Demonstra ser um instrumento útil na simulação de condições de total transformação ou relativa homogeneidade dos tecidos urbanos, assim como no auxílio ao planejamento urbano, não determinando, entretanto, o que deve ser feito, mas sim, possibilitando entender o que está sendo feito.
\end{abstract}

PALAVRAS-CHAVE: Planejamento Urbano. Acessibilidade Urbana. Mobilidade Urbana

\section{PREDICTION MODEL ACCESSIBILITY AND URBAN MOBILITY}

\begin{abstract}
Present the results obtained with the development of the Sustainable Cities Displacement Model, indicate the possibilities of continuing the research and contribute suggestions for future studies are the objectives of this article. It is a prediction model that allows comprehensive analysis of the movement of pedestrians and vehicles, as alternative simulates urban design decisions and verifies the influence of urban planning systems in new and existing areas. Operationally feasible and promising, the mathematical model shows significant statistical results with a high degree of reliability. Proves to be a useful tool to simulate conditions of total transformation or relative homogeneity of the urban systems, as well as aid to urban planning, not determining, however, what should be done, but, allowing understand what is being done.
\end{abstract}

KEY-WORDS: Urban Planning. Urban Accessibility. Urban Mobility

\footnotetext{
1 Doutor em Planejamento Urbano e Regional - UFRGS; Arquiteto e Urbanista - UNISINOS, Professor do curso de Graduação e Pós-graduação em Arquitetura e Urbanismo - UNISINOS; Pesquisador CNPQ/CAPES. e-mail: silandre@unisinos.br
} 


\section{MODELO DEL PREDICCIÓN DE LA ACCESIBILIDAD Y MOVILIDAD URBANA}

\section{RESUMEN}

Presentar los resultados obtenidos con el desarrollo del modelo del predicción de La accesibilidad y movilidad urbana, indicar las posibilidades de continuar la investigación y contribuir sugerencias para futuros estudios son los objetivos de este artículo. Se trata de un modelo de predicción que permite el análisis integral del movimiento de peatones y vehículos, como simula alternativas decisiones de diseño urbano y verifica la influencia de los sistemas de planificación urbana en áreas nuevas y existentes. Operativamente viable y prometedor, el modelo matemático muestra los resultados estadísticos significativos con un alto grado de fiabilidad. Demuestra ser una herramienta útil para simular las condiciones de transformación, total o relativa homogeneidad del tejido urbano, así como la ayuda a la planificación urbana, no determinar, sin embargo, lo que se debe hacer, pero ,lo que permite entender lo que se está haciendo.

PALABRAS-CLAVE: Planificación Urbana. Accesibilidad Urbana. La movilidad urbana

\section{INTRODUÇÃO E CONTEXTUALIZAÇÃO}

Modelos urbanos ao invés de servirem como meio para explicações diretas de fatos observados, enfocam as diferentes possibilidades de relações e interações por detrás dos acontecimentos (ECHENIQUE, 1975: 13-16; ALEXANDER, 1980: 17). Empregados comparativamente, simplificam a realidade por meio da seleção das principais propriedades capazes de descrevê-la (NOVAES, 1982; SABOYA e CATTONI, 2000). A cada dia, se tornam instrumentos de uso mais frequente pelos planejadores urbanos, pois eliminam a necessidade de testes de campo onerosos e demorados que, não raras vezes, são impraticáveis. Além disso, permitem a visualização rápida e pseudo-realista das alterações morfológicas em sucessivos intervalos de tempo, fundamental quando da análise da transformação dos tecidos urbanos. Fornecem a oportunidade de testar alternativas de desenho urbano, antes da sua implementação de fato, e podem representar tanto a (des)ocupação de lotes, edificações e espaços abertos públicos em intervalos de tempo, quanto os contatos sociais (movimento de pedestres e veículos). Deste modo, é possível adquirir 
Revista Nacional de

Gerenciamento de Cidades

conhecimento sistemático do comportamento do sistema urbano, ao simular as transformações morfológicas e cogitar suas implicações sociais.

Reflexões sobre o movimento de pedestres e veículos, enquanto materialidade fundamental para a dinâmica do cotidiano, expressam a necessidade de conhecer melhor esse fenômeno de modo a subsidiar o desenho e o planejamento urbano. $\mathrm{O}$ objetivo da presente pesquisa é apresentar os resultados obtidos junto ao desenvolvimento do modelo matemático-estatístico denominado Modelo de Deslocamento Sustentável em Cidades. O modelo permite analisar o movimento de pedestres e veículos, de modo a simular alternativas de decisões de desenho urbano e verificar a influência dos regimes urbanísticos no planejamento de áreas novas e preexistentes.

\section{CONCEITO, MÉTODO E RESULTADOS}

O Modelo de Deslocamento Sustentável em Cidades é composto por representações gráficas (esquemas que capturam os atributos geométricos e relacionais das partes que formam o tecido urbano); expressões matemáticas e estatísticas (números e equações que descrevem dimensões, proporções etc); e, descrições verbais (análise e interpretações dos resultados quantitativos e dos atributos qualitativos das variáveis).

O Modelo de Deslocamento Sustentável ${ }^{2}$ em Cidades fornece um quadro simplificado e inteligível daquilo que, fundamentalmente, são as propriedades espaciais, formais e funcionais consideradas relevantes para o problema de pesquisa. Trata-se de um recorte de uma dada realidade, em que uma série de outras variáveis, cálculos matemáticos e técnicas não foram consideradas em razão das abordagens adotadas junto ao tema. Assim, a construção do método

\footnotetext{
${ }^{2}$ Cabe destacar que o termo sustentabilidade aplicado na presente pesquisa, desde o ponto de vista da mobilidade urbana, remete a tecidos urbanos ambientalmente corretos, socialmente responsáveis e economicamente viáveis.
} 
Revista Nacional de

Gerenciamento de Cidades

compatibiliza e conjuga, por meio da construção do modelo de regressão linear múltipla, com capacidade explicativa significativa, cerca de 20 variáveis do tecido urbano. A adoção destes procedimentos estatísticos permite testes sistemáticos, pois a composição do modelo e o procedimento estatístico de análise de regressão provêm uma estrutura flexível capaz de medir a influência de diferentes unidades de análise entre si. Amplamente utilizadas em análises urbanas, as análises estatísticas tratam de quantificar determinados fenômenos e verificar as relações de causa e efeito (BARBETTA, 1999: 242).

Desagregando o Modelo de Deslocamento Sustentável em Cidades para a análise da variável dependente (movimento de pedestres e movimento de veículos) obteve-se, respectivamente, os seguintes coeficientes de regressão (tabela 1) e expressões matemáticas (quadro 1) dos maiores coeficientes de determinação estatística.

Tabela 1 : Matriz do coeficiente de regressão

\begin{tabular}{c|c|c|c}
\hline Variável independente (x) & Variável dependente $(\mathbf{y})$ & $\begin{array}{c}\mathbf{r}^{2} \\
\text { Determ. }\end{array}$ & $\begin{array}{c}\propto \\
\text { Sig. }\end{array}$ \\
\hline Modelo de Deslocamento & Movimento de pdst + vcl/100m/5min & 0,8552 & $3,58 \mathrm{E}-18$ \\
\cline { 2 - 4 } $\begin{array}{c}\text { Sustentável em Cidades } \\
\text { Integração Global }\end{array}$ & Movimento de pedestres/100m/5min* & 0,7852 & $2,73 \mathrm{E}-14$ \\
\cline { 2 - 4 } & Movimento de veículos/100m/5min* & 0,8368 & $6,2 \mathrm{E}-17$ \\
\hline Modelo de Deslocamento & Movimento de pdst + vcl/100m & 0,8543 & $4,1 \mathrm{E}-18$ \\
\cline { 2 - 4 } $\begin{array}{c}\text { Sustentável em Cidades } \\
\text { Integração Local_R4 }\end{array}$ & Movimento de pedestres/100m5min* & 0,7796 & $4,91 \mathrm{EE}-14$ \\
\cline { 2 - 4 } & Movimento de veículos/100m5min* & 0,8365 & $5,61 \mathrm{E}-17$ \\
\hline
\end{tabular}

*dados normalizados para raiz quarta ( $\left.\mathrm{A}^{-}\right)$. A abreviatura 'pdst + vcl' significa a soma do movimento de pedestres e veículos. Onde $\mathbf{r}^{2}$ é o coeficiente de determinação $\propto$ sig é a significância estatística.

(fonte: obtido no software Excel, MiniTab; original do autor, 2015)

Obtém-se o valor do Modelo de deslocamento Sustentável em Cidades a partir dos seguintes dados [(eq. 1; eq. 2; eq. 3); qd. 1] e expressões matemáticas e estatísticas com maiores coeficientes de determinação das regressões encontradas. 
Revista Nacional de

Gerenciamento de Cidades

\section{Quadro 1: Expressões matemáticas do Modelo de Deslocamento Sustentável em Cidades}

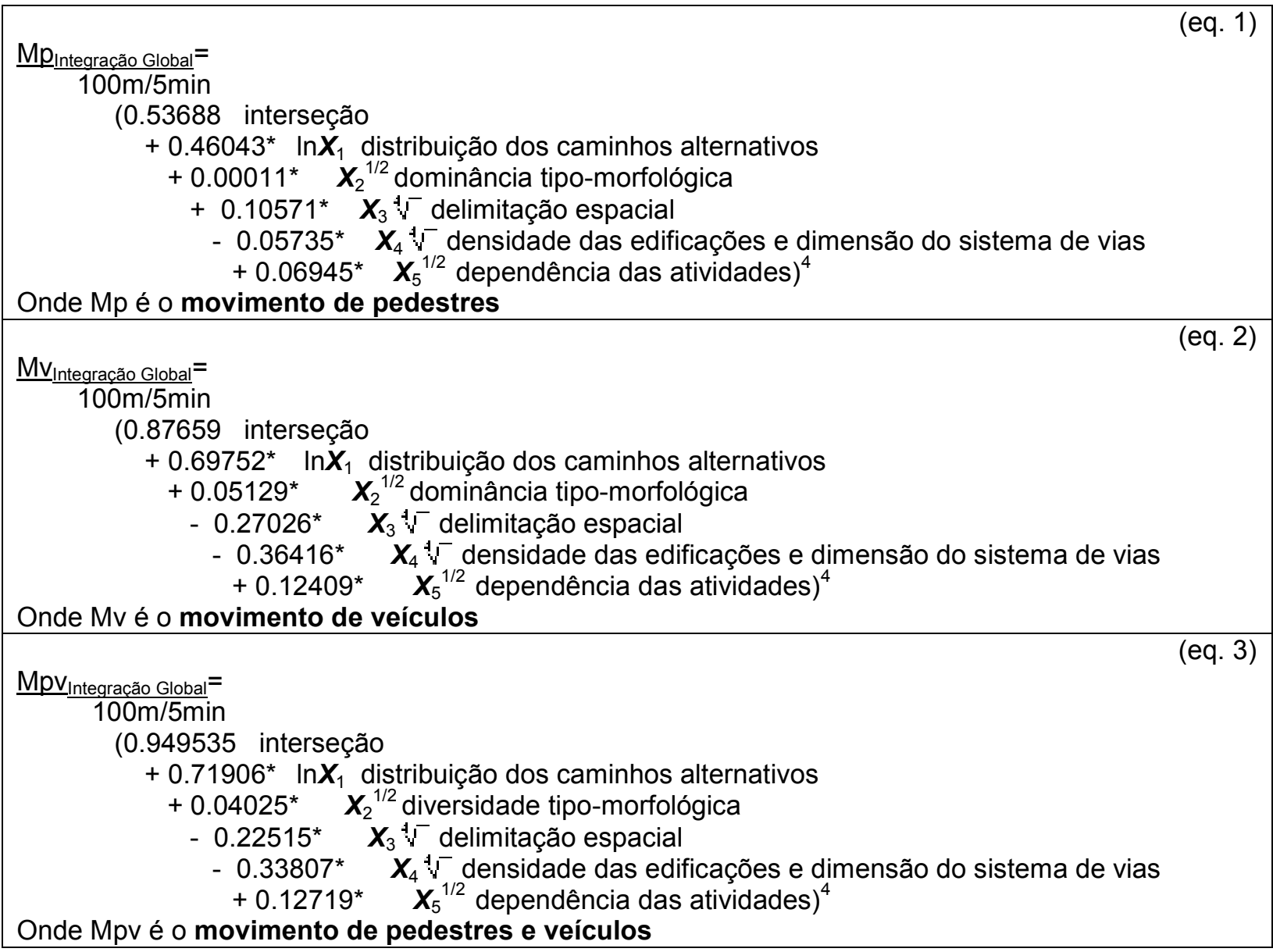

(fonte: obtido no software Excel, MiniTab; original do autor, 2015).

Uma vez determinados os parâmetros das equações, estas podem ser utilizadas para estimar o movimento de pedestres e veículos, feita através da substituição dos valores de $X 1, X 2, X 3, X 4$ e $X 5$ nas respectivas expressões matemáticas das equações com maiores coeficientes de regressão.

O desenvolvimento e aplicação do Modelo de Deslocamento Sustentável em Cidades possibilita descrever a condição do cenário atual, em termos de uso e ocupação do solo, e o respectivo efeito quantitativo no movimento de pedestres e veículos. Demonstra também ser possível predizer os efeitos tanto do cenário legal 
Revista Nacional de

Gerenciamento de Cidades

ditados por normativas prescritas nos planos diretores (índices, taxas e coeficientes), quanto o cenário proposto das alternativas de desenho urbano. As análises permitem compreender o tecido urbano em estudo e determinar as variáveis relevantes, bem como a natureza de suas inter-relações. Problemas existentes (cenário atual), assim como problemas futuros (cenário legal e proposto) - sob o ponto de vista dos efeitos no movimento de pedestres e veículos-, são detectados com base na avaliação e seleção de alternativas para a tomada de decisão em planejamento urbano (SILVA, 2010).

O Modelo de Deslocamento Sustentável em Cidades mostra-se operacionalmente viável e promissor, apresentando resultados estatísticos significativos e com alto grau de confiabilidade. Aproximadamente, $85 \%$ do movimento de pedestres e veículos nas áreas em estudo ${ }^{3}$ (bairros Higienópolis, Santa Maria Goretti, Passo D'Areia e Vila do $\mid \mathrm{API}^{4}$ de Porto Alegre, ver figura 01) foi explicado por medidas vinculadas (i) a articulação e continuidade de percursos alternativos possíveis ao longo de uma via em relação as demais vias em seu entorno imediato; (ii) a contribuição das edificações e dos recuos laterais e frontais em relação aos lotes e quadras na conformação do espaço aberto público; (iii) a variedade de tipologias edilícias e possibilidades de combinação entre si e ocorrência de morfologias simultaneamente; (iv) a relação entre movimento, área construída e comprimento de via; (v) a atribuição de valores às diferentes atividades residenciais e não-residenciais desempenhadas nas edificações; e, (vi) a ponderação dos acessos às edificações.

\footnotetext{
${ }^{3}$ As áreas em estudo conformam um mesmo sistema urbano, i.e., possuem relação de proximidade, continuidade e articulação do sistema de vias, porém com níveis diferenciados de acessibilidade, mobilidade, densidade e diversidade de edificações e de atividades residenciais e não-residenciais. A representatividade destas áreas em termos da acessibilidade do sistema de vias, peculiaridades de desenho urbano, quantidade e diversidade de formas edificadas, concentração e distribuição de atividades residenciais e não-residenciais, possibilita operacionalizar os métodos adotados, responder o problema proposto, e testar as hipóteses levantadas.

"IAPI é a sigla de "Instituto de Aposentadoria e Pensões dos Industriários", instituição criada durante o primeiro governo Getúlio Vargas, que, entre outros fatos, teve fundamental importância na criação de um conjunto residencial para uma classe operária e urbana, estreitamente relacionada ao processo de industrialização no Brasil. Ressalte-se que o IAPI é uma Vila pertencente ao Bairro Passo D'Areia.
} 


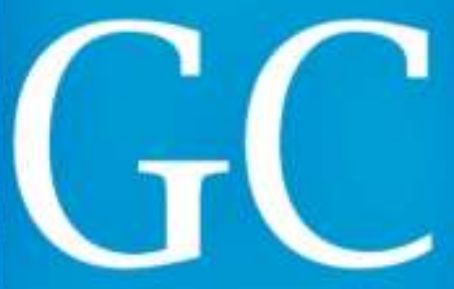

Revista Nacional de Gerenciamento de Cidades

Figura 1: Localização das áreas de estudo

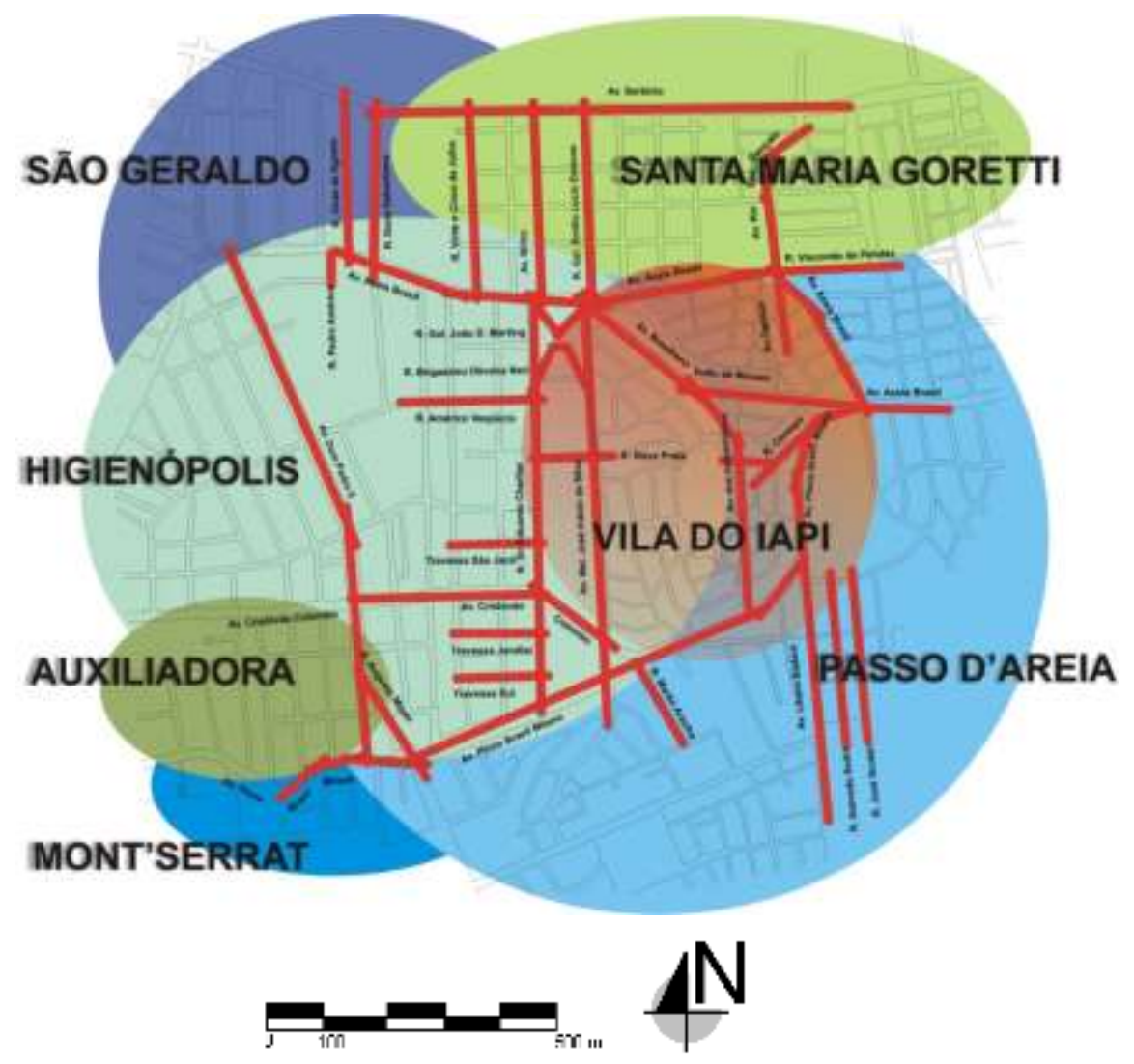

(fonte: original do autor, 2015)

A aplicação do Modelo de Deslocamento Sustentável em Cidades demonstra ser um instrumento útil na simulação de condições de total transformação ou relativa homogeneidade dos tecidos urbanos, assim como auxiliar ao planejamento urbano. As vantagens que podem ser enunciadas consistem no grau de abstração e abrangência das equações matemáticas desenvolvidas junto às medidas do tecido urbano e da necessidade de poucos dados observados, cujo tratamento estatístico comprovou determinadas correspondências entre as medidas adotadas. Soma-se a isso, o fato de que o estudo de caso nos bairros Higienópolis, $\mathrm{St}^{\mathrm{a}} \mathrm{M}^{\mathrm{a}}$ Goretti, Passo D'Areia e Vila do IAPI reforça a exequibilidade e adequação do instrumental proposto para investigar o movimento de pedestres e veículos. Contudo, faz-se 
Revista Nacional de

Gerenciamento de Cidades

necessário avaliar sistematicamente as técnicas adotadas, a fim de conduzir a um avanço metodológico, estabelecendo critérios mais adequados, e descartando outros julgados desnecessários.

Entre as demandas contempladas com o Modelo de Deslocamento Sustentável em Cidades citam-se: analisar os efeitos probabilísticos (nãodeterminísticos) que os tecidos urbanos exercem junto ao movimento de pedestres e veículos e a aplicação prática das informações obtidas; produzir informação na forma de banco de dados, de modo a gerar conhecimento sistematizado sobre o ambiente construído; otimizar o desenvolvimento de novas diretrizes para projetos futuros realimentando o processo de produção e uso; prover soluções em tecnologia da informação, garantindo a sua contínua modernização e eficiência no planejamento da mobilidade urbana; entendimento da dinâmica espacial do movimento de pedestres e veículos - com base nos resultados de simulação computacional - e suas implicações para o planejamento urbano.

Somam-se a essas metas a expectativa de que o Modelo de Deslocamento Sustentável em Cidades incentive gestores e planejadores urbanos a requalificar áreas urbanas existentes; diminua a dependência do uso do automóvel; promova o deslocamento de pedestres e bicicletas; melhore a qualidade do ar, de modo a (re)qualificar tecidos urbanos e por conseqüência, tornar as cidades mais eficientes e sustentáveis.

\section{CALIBRAGEM DO MODELO}

Calibragem consiste em buscar valores paramétricos que se ajustem o quanto possível à realidade que se pretende representar. Reif (1973: 170-172) considera três métodos de calibragem: analítico (baseado em análise estatística), heurístico (fundamentado em juízo de valor) e humano (baseado em conhecimento e observações empíricas). Batty (1976) também trata de vários métodos para a calibragem de modelos de interação espacial: por tentativas, por regressão, por 
Revista Nacional de

Gerenciamento de Cidades

estimação estatística, entre outros. Assim sendo, a calibragem sistemática das medidas do tecido urbano utilizadas, em intervalos de tempo relativamente curtos, evita que alterações no comportamento do sistema deixem de ser detectadas, gerando consequentemente distorções nos resultados.

A regressão linear múltipla utilizada como ferramenta neste trabalho é apenas um dos métodos de se quantificar as relações entre as variáveis, restando as regressões exponenciais, polinomiais, logarítmicas, dentre outras. Vale destacar que a análise de regressão não-linear apresentou coeficientes explicativos aproximados aos de regressão linear ( $r^{2} 0,8141$; sig. 1.04 E -15 para Integração Global), indicando que podem haver outros modelos que melhor respondam as medidas do tecido urbano.

As soluções analíticas e metodológicas utilizadas ainda necessitam de um maior número de estudos de caso para que possam ser comparadas e aprimoradas. Seria útil se houvesse a possibilidade do método ser gradativamente aperfeiçoado para que fossem simulados os efeitos de modificações introduzidas na composição do Modelo de Deslocamento Sustentável em Cidades. Isto certamente auxiliaria na identificação e solução de possíveis deficiências nos métodos e na validação estatística de algumas medidas do tecido urbano adotadas, possibilitando a remoção e/ou inclusão de outras medidas.

A praticidade e a operacionalidade do Modelo de Deslocamento Sustentável em Cidades revela ser possível sua aplicação em outros estudos de caso, permitindo extrapolações de grande alcance, uma vez que o movimento de pedestres e veículos apresenta certas recorrências que possibilitam a mensuração e a generalização dos resultados. Demonstra que as relações complexas do movimento de pedestres e veículos só podem ser entendidas pelas interações entre os vários elementos que compõe a demanda dos deslocamentos e os condicionantes intervenientes. Ou seja, estabelece análise de outras áreas de estudo, de modo a permitir diagnósticos comparativos entre tecidos urbanos de diferentes dimensões e características. 
Revista Nacional de

Gerenciamento de Cidades

\section{Convém ressaltar que o Modelo de Deslocamento Sustentável em Cidades} não pode ser utilizado indiscriminadamente para qualquer finalidade. Pelo contrário, sua aplicação se justifica somente ao fim para o qual foi elaborado, ou seja, é um instrumento específico para medir o quanto propriedades espaciais, formais e funcionais de tecidos urbanos influenciam no movimento de pedestres e veículos. Possíveis desdobramentos desta pesquisa podem incluir a sua utilização em áreas centrais de tecidos tradicionais, condomínios horizontais fechados, assentamentos irregulares, desde que se verifiquem e se calibrem as respectivas medidas do tecido urbano, pois nestes casos, podem ser outros os condicionantes que afetam o movimento de pedestres e veículos.

Dentro das medidas do tecido urbano que compõe o Modelo de Deslocamento Sustentável em Cidades está o levantamento e aprofundamento das possibilidades de continuidade e aprimoramento em torno de algumas variáveis consideradas, como por exemplo, a acessibilidade e a mobilidade. Foram consideradas tão-somente as características de acessibilidade ${ }^{5}$ sintática $^{6}$, mais relacionadas com a distância métrica e topológica, barreiras e passagens. Existe ainda a acessibilidade e mobilidade ditada pelas condições do sistema de vias, tais como a largura e inclinação das vias, sentido do trânsito, sinalização etc, que podem

\footnotetext{
${ }^{5}$ No âmbito desta pesquisa, em termos configuracionais, acessibilidade corresponde ao potencial de alcance das atividades e de interação das pessoas em decorrência do posicionamento relativo estabelecido entre diferentes espaços abertos públicos.

${ }^{6}$ Para medir a acessibilidade sintática por meio da integração do sistema de vias, Hillier e Hanson (1984) substituem a medida métrica pela distância topológica. A distância em Sintaxe Espacial é chamada de profundidade a qual consiste na distância topológica de um espaço a todos os demais espaços do sistema. Relações de profundidade necessariamente envolvem a noção de que o espaço aberto público pode ser acessado a partir de outros espaços adjacentes a este (HILLIER \& HANSON, 1984: 82-102). Topologia é uma condição relacional entre partes na qual independem forma, tamanho e distância métrica, e sim da articulação entre as partes, enquanto a geometria é a descrição dos elementos físicos em relação às suas dimensões, proporções, escalas etc.

“(...) Note-se que essa 'distância' é de natureza antes topológica do que geométrica, ou seja, é obtida em razão de quantas linhas, abstraídas do sistema de espaços abertos, temos minimamente de percorrer para ir de uma dada posição, na cidade, a outra posição, e não em virtude dos metros lineares de percurso que separam minimamente essas posições. Em outras palavras, num sistema muito integrado, temos de dobrar um número pequeno de esquinas para ir, em média, de uma rua para qualquer outra do lugar. O contrário se dá num sistema menos integrado (...)" (HOLANDA, 2002: 103).
} 
Revista Nacional de

Gerenciamento de Cidades

vir a ser consideradas estatisticamente significativas na composição junto ao Modelo de Deslocamento Sustentável em Cidades, possibilitando em termos metodológicos um vasto campo de investigação.

\section{PERSPECTIVAS DE CONTINUIDADE DA PESQUISA}

Ao concluir a pesquisa sobre Modelo de Deslocamento Sustentável em Cidades, novos pontos instigantes surgiram, e que de certo modo representam uma real possibilidade de continuidade da pesquisa atual. O tema enfocado e o tipo de instrumental utilizado são amplos e férteis, o que pode vir a encorajar futuros estudos. Dentre os pontos que merecem ser investigados, destacam-se:

Considera-se necessário transformar o Modelo de Deslocamento Sustentável em Cidades num software ${ }^{7}$, o qual possibilitará a geração de rotinas operacionais de acordo com a análise espacial pretendida. Operações como classificação, interpolação e sobreposição de variáveis se mostram úteis, pois são capazes de oferecer um nível de compreensão mais específico na entrada, armazenamento, transformação, análise e representação de dados. Espera-se que, ao suscitar o aprimoramento do Modelo de Deslocamento Sustentável em Cidades, seja desenvolvido um software prático, de fácil aplicação e interpretação, operacionalmente viável, capaz de aperfeiçoar a compilação, tabulação, mensuração e visualização rápida e eficiente de dados e informações necessários para analisar o fenômeno da mobilidade urbana. Soma-se a isso, o fato de que o software Modelo de Deslocamento Sustentável em Cidades pode vir a cruzar imagens diretamente da base de dados do Google_earth e Street View, e dados cadastrais técnicos multifinalitários ${ }^{8}$ (como por exemplo a PROCEMPA $^{9}$ ) e levantamento de dados diretamente no local

\footnotetext{
${ }^{7}$ Pesquisa apoiada pelo CNPQ - UNIVERSAL- MCTI/CNPq No 14/2014. Processo nº 446332/2014-3.

${ }^{8}$ Cadastro técnico multifinalitário consiste num “(...) sistema de registro dos elementos espaciais que representam a estrutura urbana ou rural, constituído por uma componente geométrica (espacial) e
} 
Revista Nacional de

Gerenciamento de Cidades

Explorar e otimizar mais o uso do sistema de informação geográfica a fim de aumentar a qualidade de visualização e precisão dos resultados para as análises espaciais urbanas. A integração com sistemas de informação geográfica possibilita a geração de rotinas de acordo com a análise espacial pretendida. Operações e rotinas como classificação, interpolação e sobreposição de mapas temáticos se mostram úteis, pois são capazes de oferecer um nível de compreensão mais específico na entrada, armazenamento, transformação, análise e representação de dados. Ou seja, aprimorar o Modelo de deslocamento Sustentável em Cidades como um instrumento prático e que fornece respostas com rapidez e eficiência.

\section{PARA NÃO CONCLUIR}

As descobertas da pesquisa sobre o Modelo de Deslocamento Sustentável em Cidades levantaram os aspectos julgados mais significativos de acordo com o recorte adotado, desde a delimitação do tema até a escolha das abordagens na demanda de viagens e na configuração espacial urbana. O Modelo de Deslocamento Sustentável em Cidades demonstra ser um instrumento útil na simulação de condições de total transformação ou relativa homogeneidade espacial, formal e funcional dos tecidos urbanos, assim como auxiliar ao planejamento urbano, não determinando, entretanto, o que deve ser feito, mas sim, possibilitando entender o que está sendo feito.

Diante do que se expôs, faz-se necessário que as pesquisas tecnológicas que apresentam resultados estatísticos significativos e com alto grau de confiabilidade sejam continuamente aplicadas e aperfeiçoadas, para que novos estudos possam ser fundamentalmente exeqüíveis e capazes de gerar resultados confiáveis ao entendimento da dinâmica espacial da mobilidade urbana. O Modelo de Deslocamento Sustentável em Cidades necessita ser continuamente divulgado,

outra descritiva que the conferem agilidade e diversidade no fornecimento de dados para atender diferentes funções, inclusive a de planejamento" (BLACHUT et al, 1974).

${ }^{9}$ PROCEMPA - Companhia de Processamento de Dados do Município de Porto Alegre. 


\section{Revista Nacional de}

Gerenciamento de Cidades

aplicado e aperfeiçoado, para que novos estudos possam ser fundamentalmente exequíveis e capazes de gerar sempre resultados confiáveis ao entendimento da dinâmica espacial do movimento de pedestres e veículos.

\section{REFERÊNCIAS}

ALEXANDER, C. La estructura del medio ambiente. Barcelona: Tusquets, 1980.

BARBETTA, P. A. Estatística aplicada às ciências sociais. Florianópolis: EdUFSC, 1999.

BLACHUT, T. J. et. al. Cadastre: various function characteristics and the planning of land Records systems. Canada: National Council Canada, 1974.

CHADWICK, G. F. Una vision sistemica del planeamiento. Barcelona: Gustavo Gilli, 1973.

ECHENIQUE, M. El concepto de sistemas, modelos y teorías en los estudios urbanos. In: Modelos Matemáticos de la estructura espacial urbana: Aplicaciones en América Latina - Buenos Aires: Ediciones Siap, (pp.: 13-46), 1975.

FOINA, P. R. Tecnologia de informação: planejamento e gestão. São Paulo: Atlas, 2001.

LEE, C. Models in planning: an introduction to the use of quantitative models in planning. Oxford: Pergamon Press, 1973.

NOVAES, A G. Modelos em planejamento urbano e regional e de transportes. São Paulo: Edgard Blücher, 1982.

HILLIER, B., HANSON, J. The social logic of space. Cambridge: Cambridge University Press, 1984.

HILLIER, B., HANSON, J., GRAHAM, H. Ideas are in things: an application of the space syntax method to discovering house genotypes. In: Environment and Planning B: Planning and Desing. London: Pion. Vol. 14. (pp.: 363 - 385), 1987.

HILLIER, B., BURDETT, R., PEPONIS, J., PENN, A. Creating Life: Or, Does Architecture Determine Anything? Architecture and Behavior 3 (pp.: 233-250), 1987.

HILLIER, B., HANSON, J., PENN, A., GRAJEWSKI, T., XU, J. Natural Movement: or configuration and attraction in the pedestrian movement urban. In: Environment and Planning $B$ : Planning and Design. London: Pion. Vol. 20. (pp.: 29 - 66), 1993.

RIGATTI, D. Do espaço projetado ao espaço vivido: modelos de morfologia urbana no conjunto Rubem Berta. Tese de Doutorado em Estruturas Ambientais Urbanas. São Paulo: Faculdade de Arquitetura e Urbanismo, Universidade de São Paulo - USP, [Disponível na Biblioteca da Faculdade de Arquitetura da UFRGS], 1997. 
Revista Nacional de

Gerenciamento de Cidades

SABOYA, R. T., CATTONI, E. L. A utilização do cadastro para análises urbanas avançadas em um Sistema de Informações Geográficas. In: Anais do IV Congresso Brasileiro de Cadastro Técnico Multifinalitário - COBRAC. Florianópolis: Cd ROM, 2000.

Disponível em <http://geodesia.ufsc.br/Geodesiaonline/ARQUIVO/COBRAC 2000/259/259.htm> Acessado em 12 de abril de 2004.

SILVA, André de Souza. Modelagem, mensuração e simulação do movimento de pedestres e veículos. Tese (doutorado) - Universidade Federal do Rio Grande do Sul, PROPUR, Porto Alegre, RS, 2010. 